\title{
Idarucizumab for Reversion of Anticoagulant Effect in Daily Practice
}

\author{
Laurent Fauchier $^{1}$ Tatjana Potpara ${ }^{2,3}$ \\ ${ }^{1}$ Service de Cardiologie, Centre Hospitalier Universitaire Trousseau et \\ Faculté de Médecine, Université François Rabelais, Tours, France \\ ${ }^{2}$ School of Medicine, University of Belgrade, Belgrade, Serbia \\ ${ }^{3}$ Cardiology Clinic, Clinical Centre of Serbia, Belgrade, Serbia
}

Thromb Haemost 2020;120:5-7.

The safety and efficacy of nonvitamin $\mathrm{K}$ antagonist oral anticoagulants (NOACs) seen in the respective pivotal randomized trials on stroke prevention in atrial fibrillation $(\mathrm{AF})^{1}$ have been broadly confirmed by numerous observational cohort- or registry-based reports. ${ }^{2-4}$ In addition to the convenient practical aspects favouring NOACs over vitamin K antagonists (e.g., no need for continuous laboratory monitoring, limited drug-drug and food interactions, fixed-dose regimen), the availability of specific antidote(s) to reverse their anticoagulant effect has been conditioned for a more widespread and possibly safer use of NOACs. ${ }^{5,6}$ Several antidotes/reversal agents for NOACs have been recently developed. ${ }^{7}$ Idarucizumab, a specific reversal agent for dabigatran, has been the first to receive approval for clinical use in many countries.

The efficacy and safety of idarucizumab for reversing dabigatran anticoagulant effect has been tested in the Reversal Effects of Idarucizumab on Active Dabigatran (RE-VERSE AD) phase III study. ${ }^{8}$ In this multicentre, prospective, single-cohort study, 503 patients receiving dabigatran were enrolled to receive idarucizumab $5 \mathrm{~g}$ in two consecutive intravenous boluses. The primary efficacy endpoint was maximum percentage of dabigatran anticoagulant effect reversal within 4 hours of the second idarucizumab intravenous bolus. The patients were divided into two groups: those with an uncontrollable or life-threatening major bleeding were enrolled in group $A$ and those needing surgery or another invasive procedure requiring normal haemostasis were enrolled in group $B$. The two groups were similar regarding most baseline characteristics; diluted thrombin time and ecarin clotting time were prolonged in more than $90 \%$ of patients at study entry but normalized rapidly after the first idarucizumab infusion, with $100 \%$ reversal within 4 hours after infusion. The reversal was sustained up to 24 hours. Among patients in group A for which the assessment of bleeding cessation could have been performed, $68 \%$ had bleeding cessation within 24 hours. For the patients in group B, peri-procedural haemostasis was considered normal in 93\% patients. In the follow-up observation, 30-day mortality rates were similar between the two groups

received

August 8, 2019

accepted

August 8, 2019

Address for correspondence Laurent Fauchier, MD, Service de Cardiologie, Centre Hospitalier Universitaire Trousseau, 37044 Tours, France (e-mail: Ifau@med.univ-tours.fr).

(13.5 and $12.6 \%$ for groups A and B, respectively). Thrombotic events occurred in $4.8 \%$ of the entire cohort.

Until recently, only small case series about idarucizumab use have been reported, ${ }^{9-11}$ and a larger study of a cohort from 20 hospitals in the Netherlands analysed 88 patients (53 with severe bleeding and 35 requiring urgent surgical intervention). ${ }^{12}$ Effective haemostasis was achieved in twothirds of bleeding patients and was associated with lower mortality risk. Clinical outcomes were considered similar to those observed in the RE-VERSE AD trial, regarding recurrent bleeding, thromboembolism and a high mortality rate.

In this issue of the journal, Fanikos et al report results of the RE-VECTO study, a global post-approval, European regulatory authorities requested international surveillance program, involving hospital pharmacies in countries where idarucizumab was dispensed from August 2016 to June $2018 .{ }^{13}$ So far, this is the largest report on idarucizumab use, including 359 patients from 61 sites in Asia, European Union and North America. The study attempted to characterize the utilization of idarucizumab in 'real-world' clinical practice worldwide, including insight into the clinical profiles of patients receiving the antidote and reasons for its use.

Generally, post-marketing observational studies provide insight into the use of medications in real-world settings, including different patient populations with variable comorbidity, different clinical circumstances and various practices or health care organizations. In the RE-VECTO study, idarucizumab was used because of bleeding in 207 patients (58\%) or an emergency surgery/urgent procedure in 129 patients (36\%), and the authors concluded that the real-world usage patterns of idarucizumab globally matched those in the clinical trial setting, with only a few off-label uses. These findings would have been reassuring if the study had not had several limitations, thus providing only a limited information on real-world idarucizumab use.

First, there could be a significant selection bias. The REVECTO surveillance program was designed to collect data on drug use in a simple and effective way but, unfortunately, the

(c) 2020 Georg Thieme Verlag KG Stuttgart · New York
DOI https://doi.org/ 10.1055/s-0039-1696646. ISSN 0340-6245. 
methods of participating centres selection are unclear. Indeed, only participating sites that prescribed idarucizumab and actually entered data into the registry were included in the study, whereas more than half of the registered hospitals did not enter patients into the registry. Among them, an unknown number of sites did use idarucizumab, but for some reasons had no capacity to enter data into the registry during the period of data collection. It is conceivable that sites realizing that idarucizumab use might not have been optimal (particularly the off-label use for NOACs other than dabigatran) were less motivated to include their unsatisfying data in the registry. It is thus possible that the rates of off-label dosing (reportedly, in $3.3 \%$ of cases only $2.5 \mathrm{mg}$ of idarucizumab was used instead of $2 \times 2.5 \mathrm{mg}$ ) and off-label use (in $1.9 \%$ of cases idarucizumab was used in patients not treated with dabigatran) have been under-estimated. Also, the data entry was not monitored, and a declarative nature of centre-reported data could have affected the overall quality of information.

Indeed, using different definitions which were not possible to evaluate in the RE-VECTO, van der Wall et al found that the use of idarucizumab was inappropriate in $28 \%$ of patients, including a significant number of patients with interventions that could have been delayed or those with bleeding complications not considered uncontrollable. ${ }^{12}$

Patients in the RE-VECTO surveillance program were rather poorly characterized regarding their baseline characteristics. While the main findings in the RE-VECTO report were broadly consistent to those observed in the RE-VERSE $A D$, the patient clinical profile in the RE-VECTO program might not be similar to that in the clinical trial, thus compromising the generalizability of the RE-VECTO findings. Also, the indication for dabigatran (i.e., the prevention of stroke in AF or prevention/treatment of deep venous thrombosis/pulmonary embolism) was not available. Hence, it is difficult to evaluate the applicability of the RE-VECTO results in these different sub-groups of patients.
Interpreting clinical results for NOACs reversal agents is difficult for several reasons. These products have been evaluated in prospective multicentre open-label studies with no comparative group. ${ }^{8,14,15}$ Whereas the use of placebo would be unethical, the efficacy of prothrombin complex concentrate in the setting of active life-threatening bleeding or urgent surgery/invasive procedure is uncertain. Hence, the primary endpoint in these studies was a percentage of reversal of the anticoagulant effect of a given NOAC. Considering the complex and highly variable clinical context, the clinical outcomes also reflect the overall clinical management beyond a purely haemostatic approach, whether considering bleeding control, thrombotic events or all-cause mortality. In addition, reversal is decided in an emergency context, where actual NOAC plasma level is most often unknown, and in many patients the NOAC anticoagulant effect might actually have expired at the time of the reversal agent administration. Considering thrombotic events, beyond the abrupt interruption of anticoagulation, there is also the risk related to immobilization, inflammation, surgery and the overall context of intensive care, which makes the identification of specific role of the reversal agent in such events difficult.

Unfortunately, the RE-VECTO registry contains no followup data on the ultimate patient outcome at discharge. This and other previously mentioned limitations of the RE-VECTO study report (i.e., a high possibility of selection bias, uncertain data quality, possible 'cherry picking' effect and unknown generalizability of the results) need to be accounted for when appreciating the contribution of the Fanikos et al report to the overall body of evidence regarding the use of idarucizumab in clinical practice.

For now, the effects of NOACs reversal agents in clinical practice remain supported by a low level of evidence, and should be evaluated in more comprehensive, better designed studies. Importantly, the use of reversal agents should be limited to specific situations ( $\mathbf{- F i g . 1}$ ), after a careful consideration of

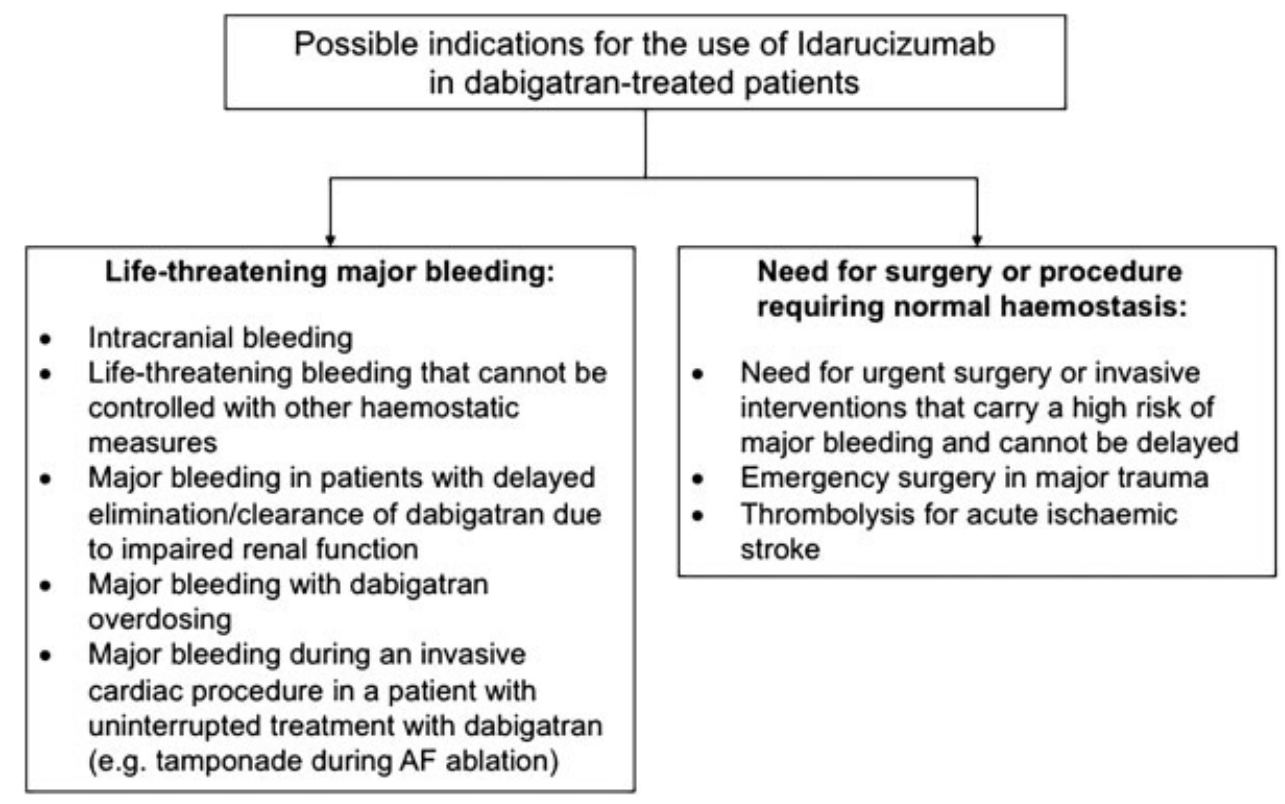

Fig. 1 Possible indications for the use of Idarucizumab in dabigatran-treated patients. AF, atrial fibrillation. 
various pharmacological and clinical issues, including alternative nonspecific haemostatic and supporting measures. ${ }^{16,17}$

\section{Funding}

None.

\section{Conflict of Interest}

L.F. has served as a consultant for Bayer, Boehringer Ingelheim, Medtronic, Novartis and Sanofi Aventis and has been on the speaker's bureau for Bayer, BMS/Pfizer, Boehringer Ingelheim, Boston Scientific and Daiichi Sankyo. T.P. has received small speaker fees from Bayer Serbia and Pfizer.

\section{References}

1 Ruff CT, Giugliano RP, Braunwald E, et al. Comparison of the efficacy and safety of new oral anticoagulants with warfarin in patients with atrial fibrillation: a meta-analysis of randomised trials. Lancet 2014;383(9921):955-962

2 Larsen TB, Skjøth F, Nielsen PB, Kjældgaard JN, Lip GY. Comparative effectiveness and safety of non-vitamin $\mathrm{K}$ antagonist oral anticoagulants and warfarin in patients with atrial fibrillation: propensity weighted nationwide cohort study. BMJ 2016;353: i3189

3 Hohnloser SH, Basic E, Hohmann C, Nabauer M. Effectiveness and safety of non-vitamin $\mathrm{K}$ oral anticoagulants in comparison to phenprocoumon: data from 61,000 patients with atrial fibrillation. Thromb Haemost 2018;118(03):526-538

4 Li XS, Deitelzweig S, Keshishian A, et al. Effectiveness and safety of apixaban versus warfarin in non-valvular atrial fibrillation patients in "real-world" clinical practice. A propensity-matched analysis of 76,940 patients. Thromb Haemost 2017;117(06): 1072-1082

5 Schulman S. New oral anticoagulant agents - general features and outcomes in subsets of patients. Thromb Haemost 2014;111(04): $575-582$

6 Lip G, Freedman B, De Caterina R, Potpara TS. Stroke prevention in atrial fibrillation: past, present and future. Comparing the guide- lines and practical decision-making. Thromb Haemost 2017;117 (07):1230-1239

7 Weitz JI, Harenberg J. New developments in anticoagulants: past, present and future. Thromb Haemost 2017;117(07):1283-1288

8 Pollack CV Jr, Reilly PA, van Ryn J, et al. Idarucizumab for dabigatran reversal - full cohort analysis. N Engl J Med 2017; 377(05):431-441

9 Vosko MR, Bocksrucker C, Drwiła R, et al. Real-life experience with the specific reversal agent idarucizumab for the management of emergency situations in dabigatran-treated patients: a series of 11 cases. J Thromb Thrombolysis 2017;43(03):306-317

10 Ebinger J, Granger CB, Zhu A, Chang A, Henry TD. Idarucizumab since FDA approval: use in the real-world. Am Heart J 2017;193:93-94

11 Brennan Y, Favaloro EJ, Pasalic L, Keenan H, Curnow J. Lessons learnt from local real-life experience with idarucizumab for the reversal of dabigatran. Intern Med J 2019;49(01):59-65

12 van der Wall SJ, van Rein N, van den Bemt B, et al. Performance of idarucizumab as antidote of dabigatran in daily clinical practice. Europace 2019;21(03):414-420

13 Fanikos J, Murwin D, Gruenenfelder F, et al. Global use of idarucizumab in clinical practice: outcomes of the RE-VECTO surveillance programme. Thromb Haemost 2019;120(01): 27-35

14 Connolly SJ, Crowther M, Eikelboom JW, et al; ANNEXA-4 Investigators. Full study report of andexanet alfa for bleeding associated with factor Xa inhibitors. N Engl J Med 2019;380(14):1326-1335

15 Pollack CV Jr, Reilly PA, Bernstein R, et al. Design and rationale for RE-VERSE AD: a phase 3 study of idarucizumab, a specific reversal agent for dabigatran. Thromb Haemost 2015;114(01):198-205

16 Niessner A, Tamargo J, Morais J, et al. Reversal strategies for nonvitamin $\mathrm{K}$ antagonist oral anticoagulants: a critical appraisal of available evidence and recommendations for clinical management-a joint position paper of the European Society of Cardiology Working Group on Cardiovascular Pharmacotherapy and European Society of Cardiology Working Group on Thrombosis. Eur Heart J 2017;38(22):1710-1716

17 Steffel J, Verhamme P, Potpara TS, et al; ESC Scientific Document Group. The 2018 European Heart Rhythm Association Practical Guide on the use of non-vitamin K antagonist oral anticoagulants in patients with atrial fibrillation: executive summary. Europace 2018;20(08):1231-1242 